\title{
Educación y naturaleza: una deuda pendiente
}

\author{
Education and Nature: A Pending Debt \\ Samantha Sparks'; Luis Corcuera-Pérez ${ }^{2}$; Ana María Vliegenthart-Arntz ${ }^{3}$
}

\begin{abstract}
RESUMEN
La Educación Ambiental al Aire Libre es una disciplina que sirve para mejorar la calidad de la educación no solo en cuanto a conocimientos y conductas ambientales, sino también para el aprendizaje en asignaturas tradicionalmente abarcadas solo en la sala de clases. En este artículo se presenta un breve marco conceptual $\mathrm{y}$ algunos fundamentos que muestran que actividades desarrolladas en contacto con la naturaleza, con la metodología propia de esta disciplina, son una fuente de motivación, aprendizajes intelectuales y desarrollo personal. La Educación Ambiental al Aire Libre responde a dos grandes imperativos de nuestros tiempos: la necesidad de formar personas que trabajan en el cuidado de la naturaleza y el insistente llamado a mejorar el proceso educativo. No obstante, un obstáculo para su implementación en el sistema formal es su magra presencia en la formación inicial docente y las pocas oportunidades de perfeccionamiento en este ámbito.
\end{abstract}

Palabras claves: educación ambiental; naturaleza; emociones; aprendizaje; formación docente.

\begin{abstract}
Nature-based education is a discipline that can serve to improve the quality of education, not just in terms of environmental knowledge and conduct, but also to promote learning in subjects traditionally taught in the classroom. We present a brief conceptual framework and argue that outdoor activities using naturebased methodology are a source of motivation, intellectual learning and personal development. Naturebased learning responds to two imperatives of our time: the necessity of forming people who will work to protect nature, and the insistent call to improve the educational process. However, an obstacle to implementing nature-based learning in the formal system is its near absence in teacher formation and the lack of training opportunities for in-service teachers.
\end{abstract}

Keywords: Environmental education; nature; emotions; learning; teacher training.

\footnotetext{
1 Directora Ejecutiva, Fundación Parque Katalpi, Puerto Montt, Chile; magíster en Ciencias Sociales; samantha.sparks@parquekatalapi.cl.

2 Presidente, Fundación Parque Katalpi, Puerto Montt, Chile; doctor en Patología Vegetal; luis.corcuera@parquekatalapi.cl.

${ }^{3}$ Vicepresidenta, Fundación Parque Katalpi, Puerto Montt, Chile; magíster en Educación, mención Currículum e Instrucción; magíster en Ciencias, mención Educación Ambiental; avliegen@parquekatalapi.cl.
} 


\section{Marco conceptual}

Los conceptos de la educación ambiental acordados por las Naciones Unidas en Tbilisi (Unesco, 1978), se mantienen vigentes y ofrecen un camino para los educadores ambientales.

La Educación Ambiental es un proceso dirigido a desarrollar una población mundial que esté consciente y preocupada del medio ambiente y de sus problemas y que tenga la motivación, los conocimientos, la actitud, las habilidades y las conductas para trabajar, ya sea individual o colectivamente, en la solución de los problemas presentes y en la prevención de los futuros (Unesco, 1978, p.27-28).

La educación ambiental se ha desarrollado como una disciplina holística, integradora y transversal a todas las asignaturas (Comisión Nacional del Medioambiente [Conama], 1993). Es participativa e inclusiva y parte con la autoconstrucción de conocimientos basada en la experiencia, por lo cual tiene pertinencia local. Otra de sus características es que reconoce el rol de las emociones en el proceso de aprendizaje y para esto se sustenta en estudios de la neurociencia sobre el proceso del aprendizaje, los cuales afirman que no existe un pensamiento puramente racional y objetivo, sino que, según sea nuestra reacción emocional con la información, esta quedará en la memoria de largo o corto plazo.

Uno de los cinco objetivos generales que establece la definición de la educación ambiental que propone la Unesco es formar personas conscientes y preocupadas por el medioambiente. Estar consciente es una experiencia que se vincula a las seis emociones básicas: el amor, la alegría, la tristeza, la rabia, la sorpresa y el miedo. Estas emociones proveen el sustento sobre el cual se construye la pedagogía para la educación de niños/as, jóvenes y personas adultas (Lenzen 2015). Estar en la naturaleza es una oportunidad para sentir varios estímulos emocionales que crean deseos de saber más respecto de aquello que nos rodea, cumpliéndose así con el primer pilar de todo proceso educativo: la motivación. Etimológicamente, emoción viene del latín emotio - onis que significa “el impulso que induce a la acción”. Siendo docentes, la acción que deseamos provocar es el deseo de aprender y para ello nada es mejor que observar cambios y procesos en la naturaleza que provocan curiosidad y asombro. Curiosidad que impulsa a profundizar en los conocimientos y 
EDUCACIÓN Y NATURALEZA: UNA DEUDA PENDIENTE / SPARKS, CORCUERA-PÉREZ, VLIEGENTHART-

ARNTZ

asombro que impulsa a la creatividad. Es aquí donde se unen los objetivos de la Educación Ambiental al Aire Libre y la búsqueda de la educación de calidad transversal.

\section{Un problema actual}

Niños, niñas, jóvenes y personas adultas de hoy viven en ciudades y pasan meses sin tocar un árbol, sin visitar un ecosistema prístino, sin respirar aire puro, muchas veces perdidos/as en mundos virtuales de pantallas. Han perdido así la capacidad de reconocer que nuestro bienestar depende del oxígeno de los árboles, los alimentos de la fertilidad del suelo, el agua pura de una cuenca lejana. Todavía tenemos naturaleza alrededor de nuestras ciudades, también en los patios de algunas escuelas; estos son los espacios que necesitamos usar urgentemente para entregar educación de calidad.

Volver a sentirnos pequeños/as, un ser más dependiente de la naturaleza, con pocas habilidades de sobrevivencia al alejarnos de la ciudad, sienta la base para un cambio de conducta de enormes proporciones. No llegaremos a respetar realmente la naturaleza hasta que aprendamos a verla como un ser vivo, animado, hasta que nos demos cuenta de que nosotros/as también somos naturaleza que camina, respira y habla; y que la naturaleza sobrevivirá sin nosotros/as; pero no nosotros/as sin ella.

\section{Los conocimientos no bastan}

En el siglo XXI contamos con un cúmulo de conocimientos y evidencia de los problemas ambientales. Hace ya medio siglo que los/as mejores científicos/as del mundo informaron a la humanidad que uno de los mayores peligros para el futuro estaba vinculado a la acumulación de dióxido de carbono $\left(\mathrm{CO}_{2}\right)$ en la atmósfera (McNutt, 2019). Sus predicciones, en cuanto al impacto de la acumulación de $\mathrm{CO}_{2}$ y al calentamiento global derivado de ello, se han ido cumpliendo tal como lo pronosticaron. No obstante, en gran medida sus recomendaciones fueron ignoradas y hoy nos enfrentamos a una grave crisis ambiental. 
En 2017 se publicó otro informe basado en los conocimientos acumulados al presente, los estudios, análisis y conclusiones de 15.364 científicos de 184 países, titulado "Segunda Advertencia a la Humanidad" (Ripple y otros, 2017). En este informe los/as científicos/as recomiendan 13 grandes cambios necesarios para que la humanidad revierta las amenazas ambientales. Doce de estos están vinculados a cambios de tecnología, en tanto solo uno se vincula a educación: aumentar la educación ambiental para niños y fomentar un mayor aprecio por la naturaleza por parte de la sociedad (2017, p. 9).

Estos/as científicos/as reconocieron que la acumulación de conocimientos, por sí sola, no es suficiente para inducir a las personas a asumir los cambios de conducta necesarios para avanzar hacia el desarrollo sustentable. Sin embargo, hasta el presente, el sistema educativo tradicional se concentra en impartir contenidos abstractos, sin las necesarias integraciones entre disciplinas y la realidad, en desmedro de una educación que tenga como centro las personas y su relación emocional con la naturaleza. Para salvar el planeta, debemos amar la tierra (Freire, 2011). La importancia del contacto con la naturaleza para desarrollar conductas ambientales respetuosas deriva del hecho de que las emociones son vitales en el desarrollo de conductas protectoras. En efecto, nada lleva más a amar la naturaleza que tener buenas experiencias, resignificar los conocimientos y saberes, y plantearse posibilidades novedosas para inscribirse en la sociedad de una manera activa. Esto se logra al acompañar la experiencia de conexión emocional con la naturaleza con buenas reflexiones de los aprendizajes logrados, las que en procesos de educación transforman una experiencia en una "experiencia significativa".

\section{El ciclo experiencial de aprendizaje}

El Ciclo Experiencial de Aprendizaje es un recurso pedagógico cuyo eje es la capacidad de aprender a partir de la propia experiencia. El desarrollo de la conciencia, que pareciera tan difícil de lograr, puede ser trabajado con actividades simples. Para niños y niñas, se ha visto que simplemente pasar tiempo en la naturaleza, jugando, explorando y descubriendo, es suficiente para estimular la curiosidad, provocar asombro y crear lazos de amor y conductas protectoras. Para jóvenes, se recomienda trabajo en equipo para explorar la naturaleza, plantear preguntas de investigación, observar, registrar y comunicar resultados, entre otras actividades. El aprendizaje 
EDUCACIÓN Y NATURALEZA: UNA DEUDA PENDIENTE / SPARKS, CORCUERA-PÉREZ, VLIEGENTHART-

ARNTZ

basado en la naturaleza también conlleva beneficios relevantes para el desempeño académico, incluyendo mejor y más eficiente funcionamiento cognitivo, mejores resultados en pruebas estandarizadas y mejoras medibles del bienestar de los/as estudiantes (Chawla, 2018). Por ejemplo, un proyecto de huerto escolar, con duración de 12 semanas, logró integrar y nivelar la participación de estudiantes diagnosticados con dificultades de aprendizaje (Schneller y otros, 2015). Otro ejemplo de cómo se están complementando metodologías de la Educación Ambiental al Aire Libre y prácticas para mejorar la educación, es el proyecto Beetles del Lawrence Hall of Science, de la Universidad de California, Berkeley, Estados Unidos (Romero y otros, 2015). Las actividades pedagógicas y formación profesional que ofrecen son una respuesta concreta a los nuevos estándares propuestos para enseñanza de la ciencia natural en ese país.

El ciclo experiencial es válido para programas de variadas disciplinas. Como primer paso el/la docente deberá considerar conocimientos previos, así como también prejuicios e inquietudes, para crear un programa educativo que se inicia con una experiencia significativa de contacto con la naturaleza.

Cuando estos programas se combinan hábilmente con actividades para el desarrollo personal, fortalecimiento de la autoestima, formación de habilidades de liderazgo y capacidades de trabajo en equipo, estamos ante la herramienta más potente que existe para el logro de educación de calidad. Cuando las actividades se realizan de manera integrada, se logran sorprendentes efectos de motivación, educación científica, artística, expresión literaria, entre otros.

\section{La educación ambiental en la formación inicial docente}

Las mallas curriculares de las carreras de pedagogía se concentran en entregar información de las especialidades de cada una (matemáticas, arte, literatura, etc.,) y en una variada gama de cursos de formación para aumentar conocimientos pedagógicos. Es notable, sin embargo, la ausencia casi total de educación ambiental como disciplina en la formación inicial docente (Vliegenthart, 2004; Vliegenthart 2018; Muñoz-Pedreros, 2014). Los equipos directivos de escuelas y los/as docentes no saben ocupar la naturaleza para cumplir los objetivos de sus asignaturas (Torres y otros, 2016). Aburridas asignaturas de aula, al ser complementadas con actividades en la naturaleza, pueden 
REVISTA SABERES EDUCATIVOS, Nº 4, ENERO-JUNIO 2020

convertirse en una motivante experiencia para generar mejores artistas, matemáticos/as, poetas, científicos/as, deportistas y un sinnúmero de profesionales motivados/as con una visión más integrada del mundo que nos rodea.

\section{Conclusión}

El contacto con la naturaleza, acompañado de buenos programas educativos, transforma simples paseos en experiencias significativas que provocan asombro, curiosidad y amor hacia la naturaleza, las cuales perduran toda la vida. Este tipo de programas también facilita lograr ciertos objetivos de aprendizaje del currículum oficial. Los centros de educación ambiental tienen un rol importante en formar y capacitar docentes. Las escuelas de formación pedagógica debieran también incluir e integrar la educación ambiental al aire libre en sus mallas curriculares. La crisis ambiental global exige un nuevo paradigma educacional basado en educación ambiental interdisciplinaria.

\section{Referencias}

Comisión Nacional del Medioambiente. (1993). Bases para una propuesta de un Plan Nacional de Educación Ambiental. Santiago, Chile: Conama, Gobierno de Chile.

Chawla, L. (2018). Nature-based learning for student achievement and ecological citizenship. Curriculum and Teaching Dialogue, (20), 25-34.

Freire, H. (2011). Educar en verde. Barcelona, España: Graó.

Lenzen, M. (2015). Feeling Our Emotions. SA Mind, (16), 14-15. DOI:

10.1038/scientificamericanmind0405-14

McNutt, M. (2019). Time's up, CO2. Science, (365), 411. 
EDUCACIÓN Y NATURALEZA: UNA DEUDA PENDIENTE / SPARKS, CORCUERA-PÉREZ, VLIEGENTHART-

ARNTZ

Muñoz-Pedreros, A. (2014). La educación ambiental en Chile, una tarea aún pendiente. Ambiente \& Sociedade, 17, 177-196.

Ripple W. J., Wolf, C., Newsome, T. M., Galetti, M., Alamgir, M., Crist, E., Mahmoud, I, y Laurance, W. F. (2017). World Scientists' Warning to Humanity: A Second Notice. BioScience, 67(12), 1026-1028. DOI: https://doi.org/10.1093/biosci/bix125.

Romero, V., Chi, B., y Snow, J. (2015). BEETLES: Better Environmental Education, Teaching, Learning, and Expertise Sharing. Final Evaluation Report: A Summary of Findings for Years 2-4: 2013. Berkeley, CA, EE.UU.: The Research Group, The Lawrence Hall of Science, University of California, Berkeley.

Schneller, A. J., Schofield, C. A., Frank, J., Hollister, E. y Mamuszka, L. (2015). Applied Environmental Education \& Communication, (14), 256 - 265. DOI: https://doi.org/10.1080/1533015X.2015.1109487

Torres Rivera, L., Benavides Peña, J., Latoja, C. J., Novoa Contreras, E. (2017). Presencia de una Educación Ambiental basada en conocimiento, actitudes y prácticas en la enseñanza de las ciencias naturales en establecimientos municipales de la ciudad de Los Ángeles, Chile. Estudios Pedagógicos, 43(3). DOI: http://dx.doi.org/10.4067/S071807052017000300018 .

Unesco (1978). Informe final, Conferencia Intergubernamental sobre Educación Ambiental en Tbilisi, 14-16 oct., 1977. P UNESCO - Paris (France).

Vliegenthart, A. M. (2004). Educación ambiental para el futuro, pedagogía ambiental para el presente. Investigaciones en Educación, 4 (4), 15-37.

Vliegenthart A. M., Corcuera, E. y Quezada, M. (2018). Capítulo 15. Educación para la conservación de la biodiversidad. En F. Pérez-Quezada y P. Rodrigo. Metodologías Aplicadas para la Conservación de la Biodiversidad en Chile (pp. 519- 548). Santiago, Chile: Universidad de Chile. 